\title{
Water at Interfaces and its Influence on the Electrical Properties of Adsorbed Films
}

\author{
Osvaldo N. Oliveira Jr. ${ }^{1}$, Antonio Riul Jr. ${ }^{2}$, and Vitor B.P. Leite ${ }^{3}$ \\ ${ }^{1}$ Instituto de Física de São Carlos, USP, CP 369, 13560-970, São Carlos, SP, Brazil \\ ${ }^{2}$ Departamento de Física, Química e Biologia, FCT-UNESP, CP 467, 19060-900, Presidente Prudente, SP, Brazil \\ ${ }^{3}$ Departamento de Física, IBILCE, Universidade Estadual Paulista, 15054-000, São J. Rio Preto, SP, Brazil
}

Received on 7 October, 2003.

\begin{abstract}
This paper discusses water at interfaces with emphasis on the electrical properties of adsorbed films. Two issues are addressed, namely adsorption of organic molecules at the air/water interface in Langmuir monolayers and the influence of adsorbed water on the electrical properties of nanostructured organic films deposited onto solid substrates. In Langmuir monolayers the focus will be on the interaction of the adsorbed molecules with the underlying water, particularly with regard to the surface potential and lateral conductance of the monolayers. It will be shown that these electrical measurements are extremely sensitive to small changes in the subphase, including trace amounts of impurities. Phase transitions due to structuring of the monolayer will be discussed at the light of theoretical models that deal with proton transfer along the monolayers. Attempts will be made to connect the interpretation at the molecular level with experimental findings from techniques such as Brewster angle microscopy and fluorescence microscopy, which provide information at the mesoscopic or microscopic scale. The gradient of the dielectric constant for water at the monolayer interface is inferred from modeling the monolayer surface potential in terms of the dipole moments of the molecules. For deposited films, the discussion will be centered on the electrical properties of nanostructured films produced with either the Langmuir-Blodgett (LB) or the layer-by-layer (LBL) methods. The strong effects from adsorbed water will be presented, with mention to sensor applications where the extreme sensitivity of the electrical properties to water is exploited and to doping of a conducting polymer induced by X-ray irradiation.
\end{abstract}

\section{Introduction}

Adsorption of organic molecules at the air/water interface has been explored since ancient times and indeed a printing technique referred to as sumi-nagashi was used in Japan thousands of years ago and Aristotle occupied himself with studying the phenomenon of the decreasing of sea roughness caused by spreading oil onto the water [1]. The scientific basis for the investigation of monolayers adsorbed from organic molecules was consolidated with Irving Langmuir in the beginning of the $20^{\text {th }}$ century [2]. Significant progress was achieved when Langmuir and Blodgett developed the methods to transfer the monolayers onto solid substrates, leading to highly organized and ultrathin films, now referred to as Langmuir-Blodgett (LB) films [3]. The LB technique was first applied to amphiphilic compounds, but it was later extended to an enormous variety of materials, including polymers and other semi-amphiphilic substances [4]. This issue has been the subject of various reviews and books [5-8]. In addition to the field of organic films adsorbed at water/air or water/oil interface, water is extremely relevant when adsorbed onto solid films. Though drying procedures may eliminate moisture leading to dry films, entrained water is found to affect strongly the film properties, which can only be removed if the film is heated to considerable temperatures.

While adsorbed water may represent a drawback for the reproducibility of electrical and other properties of the films, it may also lead to opportunities to exploit the high sensitivity of these properties to the very presence of water. Here we shall dwell upon some of the applications proposed. In this connection, we shall include not only the LB films but also the ultrathin, nanostructured organic films fabricated with the self-assembly or layer-by-layer methods [9], which also allow for control of molecular architecture. The latter are much more recent, starting with the approach suggested by Sagiv and co-workers [10] based on the chemisorption of organic molecules in a layer-by-layer fashion. The resulting self-assembled films are extremely stable and useful for several applications, but the method is limited in terms of the materials to be employed as it requires specific functional groups for the chemisorption. The layer-by-layer (LBL) method based on physical adsorption, proposed by Decher et al. [9], is more versatile and has been applied to polyelectrolytes [11], conjugated polymers [12], proteins [13] and dyes [14]. In the LBL method adsorption is basically driven by ionic attraction between oppositely charged layers, but in some cases involves secondary interactions such 
as H-bonding [15].

The organization of the paper is as follows. In Section II we provide background information on Langmuir monolayers, where selected examples from the literature are used to illustrate the electrical properties of different kinds of monolayers. Section III is devoted to theoretical models to explain the surface potential and lateral conductance of Langmuir monolayers, featuring also the implications of proton transfer and formation of a $\mathrm{H}$-bonded network at the water interface. The results for films formed with the LB or LBL techniques are discussed in Section IV, where the emphasis is in the strong sensitivity of the film electrical properties to the presence of adsorbed water. Possible applications of these nanostructured films are highlighted in this connection. Concluding remarks and prospects appear in Section $\mathrm{V}$.

\section{Monolayers at the air/water inter- face}

Langmuir monolayers are formed by spreading small quantities of amphiphilic or semi-amphiphilic materials dissolved in organic, volatile solvents onto an aqueous surface [16]. The molecules spread over the surface but will not dissolve if they contain sufficiently hydrophobic moieties. Traditional compounds for Langmuir monolayers are long-chain alkanoic acids, esters, ethers, alcohols and phospholipids where the hydrophobic aliphatic chains prevent the material from dissolving into the water. The monolayers are compressed by movable barriers that constrain the molecules until the aliphatic chains are removed from the aqueous surface, which may form well-organized arrangements. The presence of the monolayer may be detected by measuring the surface pressure, i.e. the change in surface tension of the aqueous subphase due to the monolayer. When the molecules are far apart from each other, there is negligible interaction and the surface pressure is zero. As the monolayer is compressed, distinct phases may be achieved in the surface pressure-area isotherms, which depend on the type of monolayer material. The use of semi-amphiphilic and even macromolecular materials is now well established, though a number of conditions must be met for true monolayers to be formed [4]. In addition to surface pressure isotherms, other methods have been used to characterize Langmuir monolayers, including Brewster angle and fluorescence microscopy [17], in situ UV-VIS. and FTIR spectroscopy [18], X-ray reflectivity and X-ray diffraction [19], ellipsometry [20], surface potential [21] and lateral conductance [22] measurements and surface vibrational spectroscopy [23]. An overview of the methods for characterization of Langmuir monolayers is available in Dynarowicz-Latka et al. [24].

Important for the present work are the methods for obtaining the electrical properties of the Langmuir monolayers, the most popular being the surface potential and lateral conductance measurements. Surface potential is normally measured with a Kelvin probe [25] while lateral conductance can be measured by applying a d.c. or a.c. voltage to a pair of platinum electrodes immersed into the aqueous subphase [26]. Fig. 1 shows typical isotherms for a fatty acid, where the potential is zero at large areas per molecule and becomes non-zero at a critical area that is usually twice the area of the condensed monolayer [27]. The appearance of this critical area also occurs in isotherms of lateral conductance, as shown in Fig. 1 [1], ellipsometric data [27] and UV-VIS. absorbance [28]. In Section III we shall discuss the physics behind the critical density for the measurements mentioned. There are two main causes for the surface potential to be non-zero even at large areas per molecule, the most common one being the formation of large aggregates just after spreading the monolayer. This is the case of films made from macromolecules and semi-amphiphilic compounds that are highly prone to aggregation [29]. The second cause for the non-zero potential is the existence of a strong contribution to the surface potential from the double-layer formed at the monolayer/subphase interface. Examples of negative surface potentials at large areas per molecule are the monolayers from dipalmitoyl phosphatidyl glycerol (DPPG) [30], whose surface potential isotherm is shown in Fig. 2. The surface potential depends on the dipole moments of the monolayer-forming molecules and on the double-layer, as discussed in Section III. For DPPG, in particular, a negative contribution from the double-layer causes the potential to be negative at large areas.

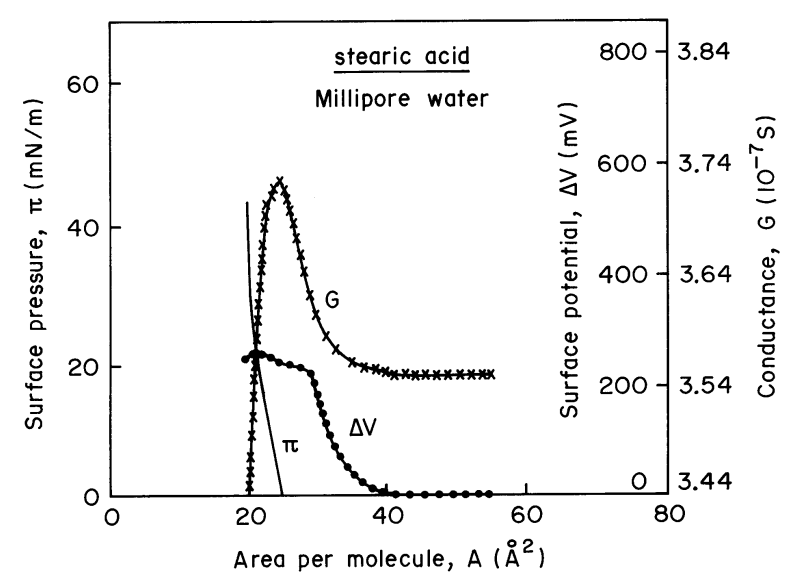

Figure 1. Surface pressure $(\pi)$, surface potential $(\Delta V)$ and lateral conductance $(\mathrm{G})$ isotherms for stearic acid. Both lateral conductance and surface potential lifts off at a critical area. The decrease in lateral conductance as the pressure starts to rise is due to the meniscus effect, by which the area of the immersed electrode decreases. This decrease is therefore an experimental artifact. From ref. [1].

The lateral conductance shown in Fig. 1 was measured by inserting two bright platinum electrodes into the aqueous subphase on which the monolayer is spread. At large areas per molecule the lateral conductance is zero, that is to say, the conductance is the same as that measured with the aqueous subphase. At the critical area the lateral conductance 


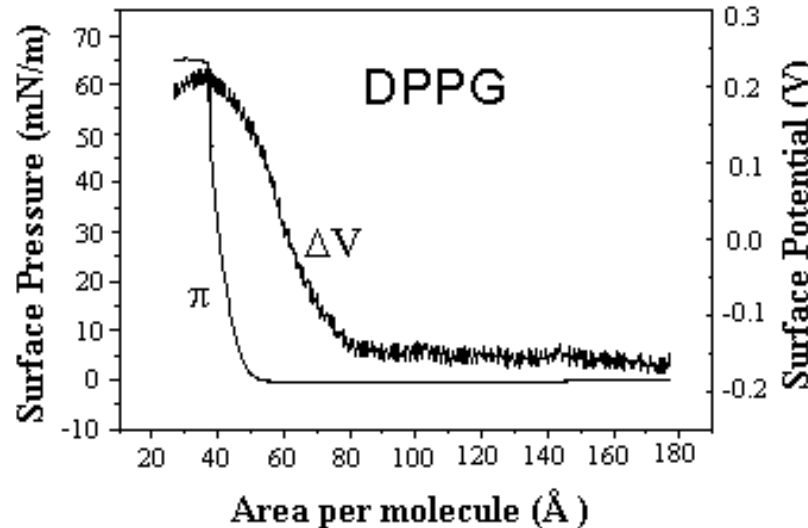

Figure 2. Surface pressure $(\pi)$ and surface potential $(\Delta \mathrm{V})$ of a DPPG monolayer spread onto ultrapure water. Note the negative surface potential, due to the contribution from the double-layer, at large areas per molecule. From ref. [30].

increases relatively sharply until it practically levels off when the monolayer is in the condensed state. If the electrodes are only partially immersed into the subphase, the increase in surface pressure causes the meniscus between the monolayer and the platinum electrodes to lower, then decreasing the measured lateral conductance [31]. This experimental artifact due to the meniscus has been well documented, but could be obviated in a special arrangement developed by Cavalli [26]. One should mention that there has been controversy in the literature over the possibility of detecting the enhanced lateral conductance, particularly because of the extremely low thickness of the monolayer. Following the conflicting reports by Morgan et al [31], Menger et al. [32] and Shapovalov and Illichev [33], Cavalli and Oliveira [34] developed a differential method to measure the conductance in which the contribution from the subphase (ultrapure water) was eliminated, thus allowing the conductance to be measured in phospholipids, alcohols and fatty acids [26]. Recent work by a Japanese group, which employed a special arrangement of electrodes [35], confirmed the possibility of measuring the lateral conductance and investigated the effects from adding alcohol to monolayers of stearic acid, elaidic acid and oleic acid. Fig. 3 shows that upon increasing the alcohol concentration the lateral conductance reaches a maximum after which it decreases. This behavior also depends on the type of acid in the monolayer, particularly with regard to the packing of the monolayerforming molecules. This is illustrated by the larger increase in conductance for a stearic acid monolayer whose molecules are more closely packed than for elaidic and oleic acid [35].

The difficulties in measuring the lateral conductance, which is indeed at the root of the controversy mentioned above, are understandable if one considers that trace amounts of impurities may affect the monolayer electrical properties significantly. The first demonstration of this statement was provided by Taylor et al [36], who compared surface potential isotherms of stearic acid spread onto ultrapure water from 2 distinct purification systems. In one case the

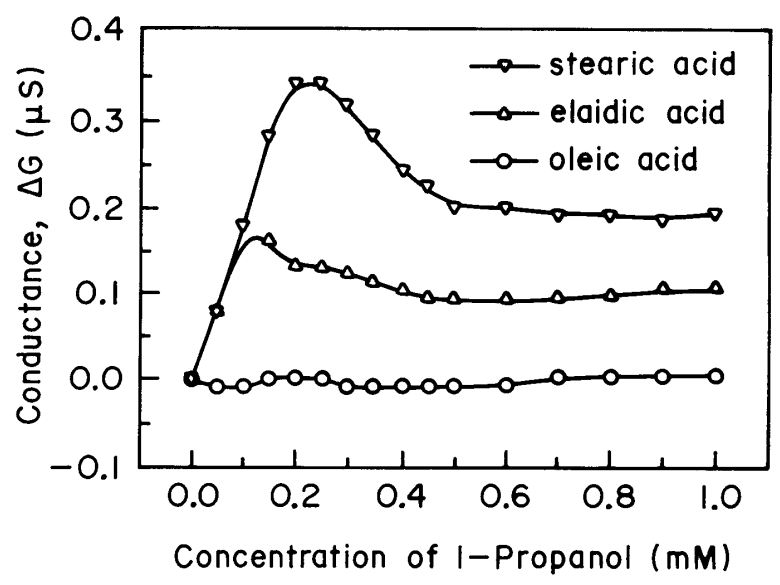

Figure 3. Conductance increase for monolayers of three acids with increasing concentration of 1-propanol. From ref. [35].

surface potential was non-zero even at large areas per molecule. In addition, reproducible results could not be obtained at large areas, which was attributed to the existence of large islands of material. A series of systematic experiments indicated that this non-reproducibility was due to trace amounts of impurities in the water subphase [36], arising from the need to store the purified water in tanks. Even though the stored water was recirculated at regular intervals, it could still be insufficient to guarantee the required purity. Modern purification systems now often provide output water directly from the polishing cartridges for purification, with the storage tank being located before the final purification stage. This is the reason why reproducible surface potential isotherms - with zero surface potential at large areas in a number of cases - have been obtained in laboratories around the world, which was not the case until the 1980s.

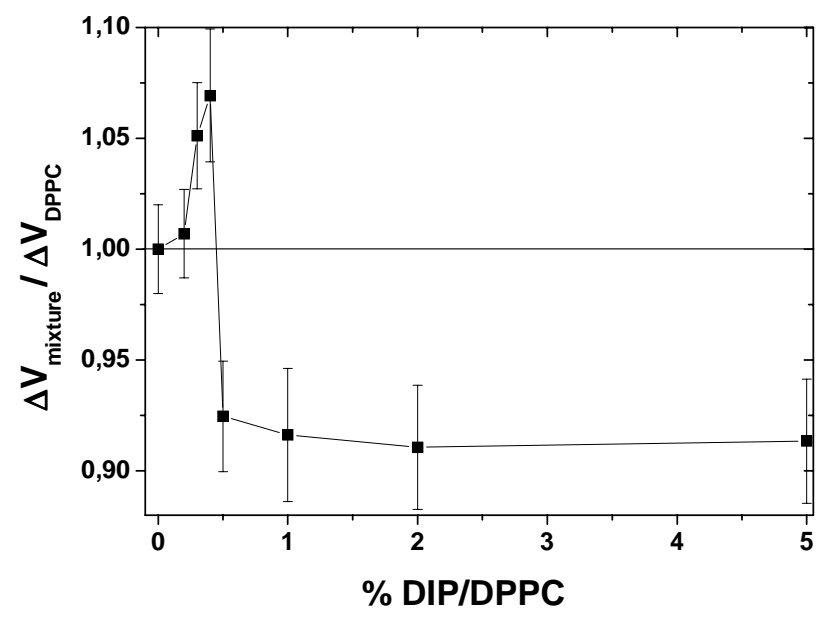

Figure 4. Ratio between the surface potential for mixed DIP/DPPC monolayers and that of a pure DPPC monolayer vs. concentration of DIP. The values were taken at the onset of the LE/LC phase transition. From ref. [37].

Further evidence of the importance of trace amounts of impurities or guest molecules for a Langmuir monolayer was provided by the cooperative response from phospholipid monolayers to pharmaceutical drugs where $0.2 \mathrm{~mol} \%$ of dipyridamole (DIP) was sufficient to alter the monolayer 
properties significantly [37]. In Fig. 4, it is seen that the surface potential of dipalmitoyl phosphatidyl choline (DPPC) varies with the concentration of DIP cospread at the air/water interface. The maximum effect appears at $0.4 \mathrm{~mol} \%$, after which the effect decreases and the surface potential becomes even lower than for pure DPPC due to self-aggregation of DIP molecules as the concentration increases. Note that for concentrations as low as $0.4 \mathrm{~mol} \%$ the changes in surface potential cannot be explained by the dipole moment of DIP molecules. Nor can they be explained by changes caused in the neighboring DPPC molecules. Therefore, these results can only be accounted for if a cooperative effect takes place, with DPPC molecules being affected which are not directly interacting with DIP molecules.

\section{Models to explain the surface po- tential and lateral conductance in monolayers}

\subsection{Models for the surface potentials}

In order to correlate the measured surface potentials with the dipole moments of the monolayer-forming molecules, theoretical models have been developed over the years in which the monolayer is treated as an arrangement of dipoles. The first of such models is referred to as the Helmholtz approach, according to which the surface potential is

$$
\Delta V=\frac{\mu_{\perp}}{\varepsilon_{0} \cdot A}
$$

where $\mu_{\perp}$ is the apparent dipole moment, which corresponds to the vertical component of the dipole moment of a molecule, $\varepsilon_{0}$ is the vacuum permittivity and $A$ is the area per molecule. The name apparent dipole moment was given because in this model the monolayer is assumed to have a unity dielectric constant, which further research has shown not to be the case. Indeed, refining of the Helmholtz model has led to 2-layer [38] or 3-layer [39] capacitor models in which

$$
\mu_{\perp}=\frac{\mu_{1}}{\varepsilon_{1}}+\frac{\mu_{2}}{\varepsilon_{2}}+\frac{\mu_{3}}{\varepsilon_{3}}=\varepsilon_{0} \cdot A \cdot \Delta V
$$

where $\mu_{1}, \mu_{2}$ and $\mu_{3}$ are, respectively, the normal components of the dipole moments of the water molecules reoriented by the presence of the monolayer, of the headgroups and of the tail groups. $\varepsilon_{i}(\mathrm{i}=1,2$ or 3$)$ are the effective dielectric constants of the media involving these dipoles. Conceptually, there is no important difference in using a 2or a 3-layer capacitor models. The 2-layer model is certainly advantageous as it has less unknown parameters, by combining the contribution from the reorientation of the water molecules with that from the headgroups. However, there are cases in which one wishes to compare results from molecules that differ only by their headgroups, and this is the main reason why the 3-layer DF model represented in
Eq. (2) has been applied to aliphatic [40] and aromatic compounds [41]. In both cases, the parameters to explain the experimental data must be taken as average values. They are practically the same for aliphatic and aromatic compounds, with the dielectric constant for the monolayer/water interface, $\varepsilon_{2}$, lying in the range between 6 and 7 and $\varepsilon_{3}$ varying from 2 to 3 [40,41]. The contribution from water reorientation is used as adjusting parameter, being slightly negative (ca. $-65 \mathrm{mD}$ ). The implication of such analysis is obvious: at water/air interfaces with an adsorbed monolayer, the effective dielectric constant changes from ca. 80 in the bulk to 2-3 at the air interface, with an intermediate value of 6-7 at the monolayer/water interface.

If the monolayer is at least partially charged, an electrical double-layer is formed at the monolayer/water interface, whose contribution to the surface potential may be estimated using the Gouy-Chapman theory [42]

$$
\Psi_{0}=\frac{2 k T}{e} \sinh ^{-1}\left[\frac{e \alpha}{A \cdot\left(5,88 \cdot 10^{-7} \cdot c \cdot \varepsilon \cdot T\right)^{1 / 2}}\right]
$$

where $k$ is the Boltzmann constant, $T$ is temperature, $e$ is the proton charge, $\alpha$ is the degree of dissociation of the headgroups, $c$ is the ionic strength of the subphase, $A$ is the area per molecule and $\varepsilon$ is the dielectric constant in the double-layer region. Implicit in the Gouy-Chapman theory are the assumptions that the charged surface is impenetrable and that the counter-ions are point charges that do not interact among themselves. In spite of these simplifications, the Gouy-Chapman theory has been successfully applied to explain surface potential data of aliphatic [43] as well as aromatic carboxylic acids [44]. There are cases where the application of Gouy-Chapman is only possible if an effective charge is assumed because counterions from the subphase tend to adsorb at the monolayer surface [45].

\subsection{Models for lateral conductance}

Pure phenomenological models can give guidelines to the understanding of interfacial systems discussed in this work. However, it is difficult to discuss lateral conductance and the gradient in the effective dielectric constant without taking into account molecular aspects of the film and the water in the interface. Treating the film/water interface at the molecular level is a very difficult task though. If one considers systems at the nanometer scale, simulations at all-atoms level are probably unreliable due to the approximations one has to make. A possible solution is to seek simplified models and try to adapt results from bulk studies to the interface problems. This is what we have done to understand the appearance of a critical area for surface potential and lateral conductance data [46]; some of the arguments are reproduced here.

One of the key issues to be addressed in molecular models is the arrangement of the film molecules with respect to each other and with respect to the water. Most of these arrangements take place through hydrogen bonds, which play 
a fundamental role in molecular association. In supramolecular chemistry the hydrogen bond is able to control and direct the structures of molecular assemblies because it is sufficiently strong and sufficiently directional. In mechanistic biology, it is of vital importance because it lies in an energy range intermediate between van der Waals interactions and covalent bonds, which allows hydrogen bonds to both associate and dissociate quickly at ambient temperatures. A simple criterion for hydrogen bond formation is when the electronegativity, as defined by Pauling [47], of an atom A relative to $\mathrm{H}$ in an $\mathrm{A}-\mathrm{H}$ bond is such as to withdraw electrons and leave the proton partially unshielded. To interact with this donor A-B bond, the acceptor B must have lone-pair electrons or polarizable $\pi$ electrons. Very strong hydrogen bonds resemble covalent bonds, while very weak hydrogen bonds are close to van der Waals forces. The majority of hydrogen bonds are distributed between these two extremes. From the theoretical perspective $\mathrm{H}$-bonds have been treated in different levels of detail, from simple dipole moments and ionization potentials [48] to full ab-initio calculations.

The hydrogen bonds in general can be regarded as the incipient state of a proton transfer, and this is true for strong and as well as for the weak bonds. However, only for strong bonds proton transfer processes occur with significant rates [50]. To explain the enhanced conduction and the appearance of a critical area, we focus on the proton transfer aspects associated with $\mathrm{H}$-bonds, particularly with respect to water. A mechanism to account for the fast proton transfer in liquid water is the sequential concerted proton transfer along the hydrogen bond network [51]. Using molecular dynamics calculations, theoretical groups have observed that proton transfer is driven by the hydrogen bond coordination and distances fluctuation caused by the hydrogen bond network rearrangement $[52,53]$. Because of the difficulties for a detailed simulation of the film/water interface, we use analogies with proton transfer in bulk water and a simple model based on hydrogen bond data and geometric constraints and a unidimensional model for proton transfer (PT) in hydrogen bonds [54]. The polar groups and the water are assumed to form strong hydrogen bonds below the critical area, thus creating a highly structured media to allow for a much faster proton transfer along the hydrogen bond network. The conductance happens via a "hop and turn" mechanism [55] with proton transfer through the hydrogen bonds. The model restricts to the consequences associated with the hydrogen bond length $R$ in which the proton transfer can effectively occur, since it is crucial to explain our experimental data. The effective potentials are characterized by parameters obtained from the physical properties of the $\mathrm{H}$ bonds. In proton transfer reactions, a shift of electron density also accompanies the nuclear transfer. A localized part of the electronic density which mainly corresponds to the bond with the proton is shifted from one nucleus to another [56]. Proton transfer is based on the assumption that the nuclei move on a much slower time scale than electrons, i.e. on Born-Oppenheimer surfaces. The two electronic states seen by the proton are associated with the reaction

$$
A-H \cdots B \leftrightarrow A \cdots H-B
$$

where the state on the left hand side has electron density localized between nucleus A and the proton (called donor) and the state on the right side has density between the proton and nucleus B (called acceptor). For simplicity a system with symmetric oxygen donor and acceptor in a line was considered $\left(O_{A}-H \cdots O_{B}\right)$. The resulting effective potential as a function of the distance $r$ of the proton from the donor $\left(\mathrm{O}_{A}\right)$ is shown in Fig. 5. The two minima represent the equilibrium positions for the proton in the donor or acceptor. The effective potential barrier height increases as the distance between the oxygens $R$ increases. This feature has been confirmed by other theoretical studies [57,58]. There is a transition from a double well system to that of a single well as $R$ is decreased, which corresponds to a strong hydrogen bond. The $O-H \cdots O$ hydrogen bond lengths in carboxylic acids and their hydrates exhibit a wide range, from very short, $2.5 \AA$, to relatively weak bonds with $R \sim 2.9$ $\AA$. According to our model, proton transfer can effectively occur when $R$ is smaller than $\sim 2.8 \AA$. We point out that in this first analysis, we just want to draw reasonable numbers to understand the experimental results.

The polar part of amphiphilic molecules in the films is arranged in a bidimensional triangular lattice. We assume that between the polar groups there are water molecules, through which a net of H-bonds is formed (Fig. 6). Also assumed is a simple geometric model for the configuration of these molecules, as illustrated in Fig. 7. The critical area for fatty acids corresponds to a distance of $7.1 \AA$ between polar heads $\left(38 \AA^{2} /\right.$ molecule). This distance corresponds to a distance $R$ between donor and acceptor oxygens of $2.8 \AA$. Below this distance a net with strong H-bonds is formed. The H-bond strength and proton transfer increase exponentially with decreasing $R$. This suggests that the dramatic increase in the conductance below the critical area is due to a proton in a "hop-and-turn" mechanism. These strong $\mathrm{H}$-bonds explain also the abrupt increase, below the critical area, in surface potential. It is associated with a drastic decrease in the dielectric constant at the film-water interface caused by structuring of the monolayer. This is the simplest first order approach to model the monolayer structuring via H-bonds. There are a number of other important factors that should be included in a detailed description. The oxygens were considered chemically equivalent, yielding symmetric potentials. The oxygens from water molecules and from the polar headgroups are not chemically equivalent, so the asymmetry should be included in a more detailed study. Recently a soliton model based on these ideas was presented [59]. It uses three fields describing: the hydrogen involved in the conduction, the hydrophilic head of the film and the water. It has established the grounds to treat the experimental data quantitatively. 


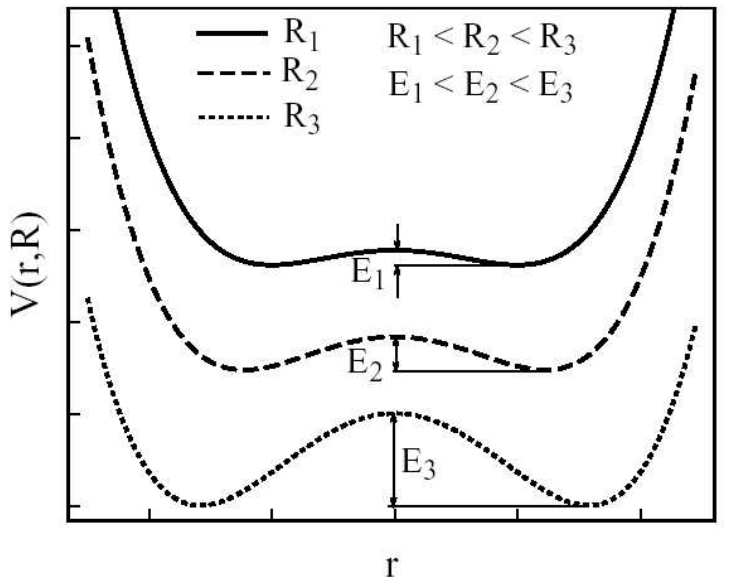

Figure 5. Qualitative behavior of the effective potential V(r,R) as a function of proton coordinate $r$ for different oxygen-oxygen distances R. From ref. [46].
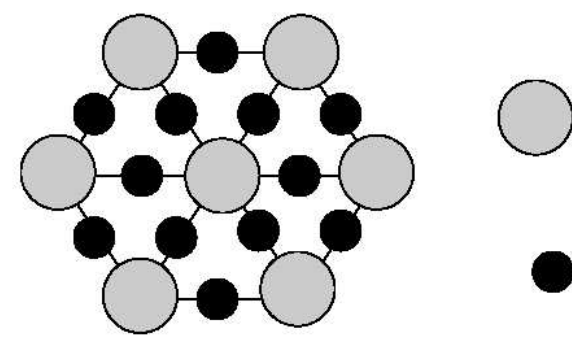

polar group

Figure 6. Bidimensional arrangement of the hydrophilic part at the monolayer/water interface. From ref. [46].

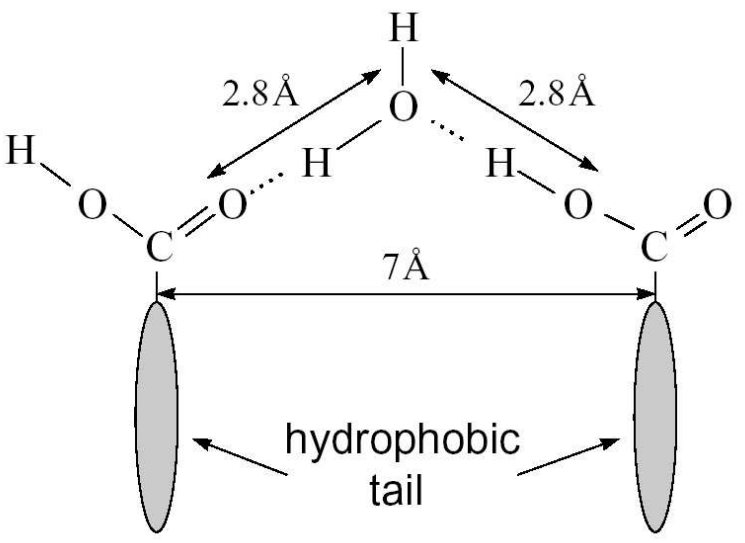

Figure 7. Geometrical arrangement necessary for the proton transfer to take place. The $\mathrm{C}-\mathrm{O}$ distance is around $1.36 \AA$ and the $\mathrm{C}=\mathrm{O}$ distance is $1.24 \AA$. The angle between $\mathrm{O}-\mathrm{C}=\mathrm{O}$ is assumed to be $120^{\circ}$ symmetrically oriented around the hydrophobic tail axis. From ref. [46].

The formation of the H-bond network is manifested at the mesoscopic or microscopic level with domains of monolayer-forming molecules achieving a state that is characterized by a critical area. The existence of this critical area has been shown not only in surface potential and lateral conductance isotherms, as discussed in the last section, but also in optical measurements such as UV-VIS. absorbance [28] and Brewster angle microscopy [60].

\section{Water adsorbed on nanostructured thin films}

The two most popular techniques to produce nanostructured films from organic molecules are the Langmuir-Blodgett (LB) and the layer-by-layer (LBL) methods. The LB technique is significantly older, dating from the mid 1930s, while the LBL method - based on physical adsorption of oppositely charged layers - appeared in the early 1990s. Both methods have been used for a variety of materials, in a number of cases leading to supramolecular structures where molecular control is made possible. Furthermore, the final film properties may differ considerably from those obtained with the same materials produced with other techniques, such as spin-coating, dip-coating or casting. Because water is involved in the fabrication process of these nanostructured films, it is not surprising that adsorbed water may play an important role. This is especially true of electrical properties, as discussed below.

\subsection{Electrical characterization of $L B$ and $L B L$ films}

Analogously to the case of Langmuir monolayers, for LB and LBL films conductivity and surface potential measurements are also the most common methods for electrical characterization. The electrical conductivity can be measured with various methods, including those allowing the measurement of surface conductance and analyzing anisotropy of the samples. For thin films, for example, the 4-point probe van der Pauw method is widely used [61]. In order to avoid artefacts from charge accumulation and high fields at the electrodes, a.c. techniques are often used with the frequency ranging from $\mu \mathrm{Hz}$ to $\mathrm{MHz}$ (this is also referred to as impedance spectroscopy). Interpretation of the data is far from straightforward, as the films are not crystalline and interactions among molecules or with the surrounding environment affect the conductivity strongly. Attempts have nevertheless been made with the use of theoretical models developed for amorphous solids [62]. For example, Bianchi et al. [63] have shown that polyaniline films may be treated as a disordered insulating matrix with conductive, doped islands, whose conductivity obeys the random free-energy barrier model [62].

The surface potential of LB or LBL films is normally measured with a Kelvin probe, with the film deposited onto a metallic substrate. The measured potential thus represents the change in work function of the metal substrate due to the film coating it. Because of the effects from adsorbed water, moisture must be eliminated, particularly for the asdeposited films, since LB and LBL films involve water in the fabrication procedure. Reproducible results are therefore only obtained if the films are left to dry for a certain period 
of time, which may vary from hours to a full day depending on the film. Interpretation of surface potentials on solid films is more involved than for Langmuir monolayers owing to the contribution from the substrate/film interface. The first attempts to model surface potentials in LB films appear to have been made by Tredgold and collaborators $[64,65]$, who considered the substrate/film interface as a p-n junction. Taking into account more recent studies, it has been established that the possible contributions to the surface potential of transferred LB or LBL films are [66]: i) dipole moments of the film-forming molecules; ii) change in the contribution from double-layer potentials as the molecules are transferred from an aqueous/air interface to a solid/air interface; iii) contribution from the substrate/film interface, which is usually due to charge injection from the metallic substrate. The surface potential for an LB monolayer can be written as [66]:

$$
V_{L B}=\Delta V_{L}+\varphi+V_{s u}
$$

where $\Delta \mathrm{V}_{L}$ is the surface potential for the non-ionized monolayer, $\varphi$ is the contribution from image charges, which essentially replaces the double-layer contribution in a Langmuir monolayer, and $\mathrm{V}_{s u}$ is the contribution from charge injection from the substrate. This expression assumes that the film molecules did not change orientation during transfer, leading to the same contribution from the dipoles as in the non-ionized monolayer. For an LB film with several layers, one has to consider the packing of the molecules. For a centrosymmetric arrangement, as in the Y-type LB films with a head-tail-head-tail organization, cancellation of dipole moments occurs. The contribution from charge injection was investigated by Iwamoto and collaborators $[67,68]$ and then determined directly by comparing surface potential data for LB films of cadmium stearate (CdSt) with different odd and even numbers of layers [66]. The interface contribution was found to be ca. $-150 \mathrm{mV}$, which was the value measured for LB films with even number of deposited CdSt layers, as the dipole contribution was cancelled [66].

\subsection{Exploiting effects from water in applica- tions}

While water at interfaces normally poses difficulties for obtaining accurate characterization of the interface properties, as mentioned above, the high sensitivity to water may be exploited in useful applications. The most obvious one is to produce humidity sensors, as demonstrated by Raposo [69] with layer-by-layer films from poly(o-methoxyaniline), and is widely reported in the literature (for a number of examples, see [70]). For instance, polyaniline films [71] have been shown to be efficient in detecting gases and vapors, including water vapors, where the principle of detection is conductivity measurements. Humidity tests carried out by exposing polyaniline samples fabricated by anodic polymerization to water vapors showed that the d.c. conductivity was altered by one order of magnitude to $1.34 \times 10^{-8} \mathrm{Scm}^{-1}$ [71]. Nanosized zeolite films [72] have also been used in piezoelectric devices based on a quartz crystal microbalance (QCM) to detect not only water but also organic compounds vapors.

However, humidity sensors may be obtained in a much cheaper way with no need to resorting to nanostructured films. There are, nevertheless, applications in which taking advantage of fine control over film thickness and molecular architecture is crucial to achieve high sensitivity. This is the case of taste sensors (so-called electronic tongues) made up from an array of sensing units composed by interdigitated gold electrodes covered with nanostructured LB or LBL films of different materials (conducting polymers, ruthenium complexes, perylenes, lipids, natural polymers) [73-76]. For the same liquid sample, the response from distinct units may be different and the combination of responses from the units comprising the array is used as a fingerprint for the sample under analysis. Normally, each array contains 4-8 units produced from materials judiciously chosen to respond in varied ways to the samples analyzed. Because of the amount of data collected for several samples with a given array, the impedance data are analyzed by taking the capacitance at a fixed frequency of the film, which is estimated from the equivalent circuit analysis. Fig. 8a shows the equivalent circuit employed, in which the film coating the electrodes is described as a parallel combination of $\mathrm{C}_{b}$ and $\mathrm{G}_{b}$. The film is assumed to be in series with the electrolyte impedance represented by the geometric capacitance of the inter electrode space filled with electrolyte $\left(\mathrm{C}_{g}\right)$ and the double-layer capacitance $\left(\mathrm{C}_{d}\right)$, which is charged from the solution conductance $\left(\mathrm{G}_{d}\right) . \mathrm{G}_{t}$ allows for charge transfer across the film/electrolyte interface [77]. The simulated response for representative values of the various parameters is shown in Fig. 8b. Following Taylor and MacDonald [77], the frequency is chosen in the range between 300 and $1 \mathrm{kHz}$, within which the electrical response is primarily driven by the film properties.

A variety of materials and liquid samples have been investigated in a project involving Embrapa (Brazil), University of São Paulo (Brazil) and the University of Wales, Bangor (U.K.). Among the main results one may include the demonstration of extremely high ability to distinguish the basic tastes, i.e. salt, sweet, sour and bitter, to a sensitivity that is in some cases 3 orders of magnitude higher than that of the human gustatory system [76]. The high sensitivity is attributed to the ultrathin nature of the nanostructured films coating the electrodes. Complex liquid systems can also be analyzed, and distinction can be made between several beverages and types of water, as illustrated in the Principal Component Analysis plot of Fig. 9 [78].

\subsection{Doping semiconducting polymers}

Doping in conjugated polymers plays an important role for the final properties of the polymer and has large impact on the possible applications. While for some conjugated polymers doping occurs via oxi-reduction reactions, for polyaniline (PANI) protonation is the basic mechanism, 
(a)
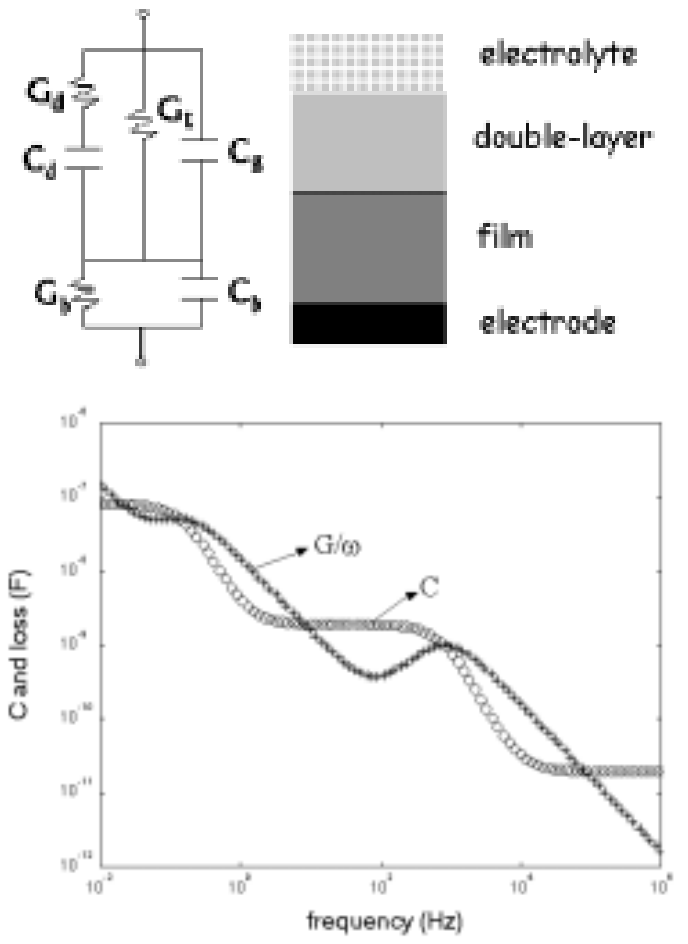

(b)

Figure 8. (a) Equivalent circuit for a metal electrode coated with a weakly conducting film; (b) Theoretical plots of capacitance (C) and dielectric loss $(\mathrm{G} / \omega)$ for the equivalent circuit illustrated (a). The values used for generating these curves are: $C_{g}=20 p F$; $C_{b}=2 n F ; C_{d}=100 n F ; G_{t}=10^{-8} S ; G_{b}=10^{-7} S$; $G_{d}=10^{-5} S$. From ref. [75].

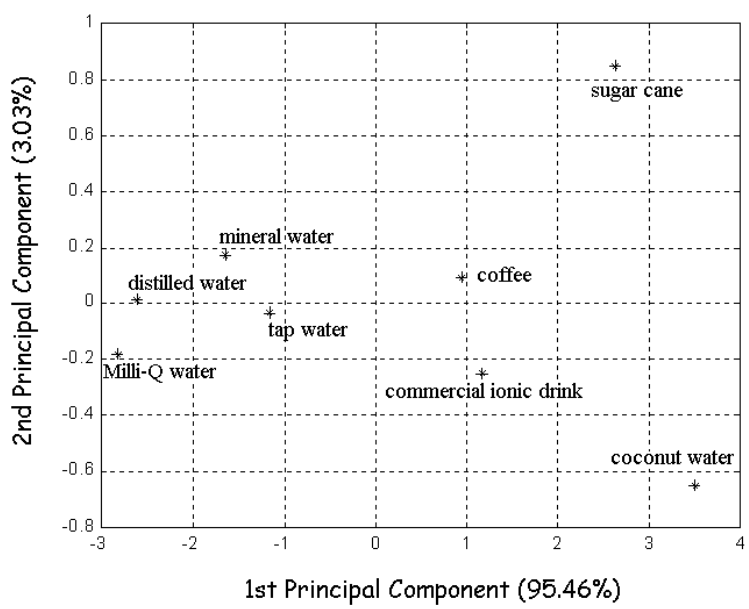

Figure 9. PCA plot of the sensor system immersed into different beverages. It is readily seen that the various samples can be distinguished from each other. From ref. [78].

which is usually carried out by exposing the polymer to acidic substances. Control of doping is expected to improve if "dry" methods are used in protonating PANI and its derivatives, especially because the electrical properties are strongly modified by residual water in the pores of the polymer matrix [79-81]. Such "dry doping" may be performed by subjecting polymer films to X-ray irradiation which causes PANI conductivity to increase by ten orders of magnitude [82], or to ultraviolet and electron beam irradiation [83]. Doping through X-ray exposure was further exploited by using LB films from PANI [84-86], where control of molecular architecture allowed a number of features to be investigated. Here we present additional data regarding this specific doping process in composite LB films of polyaniline.

The LB films were produced by transferring mixed monolayers from PANI and cadmium stearate spread on ultrapure water onto quartz and $\mathrm{CaF}_{2}$ substrates. The experimental procedures for LB film fabrication have been fully described in $[86,87]$, and only a few details are given here. PANI was synthesized according to [88]. The spreading solution was prepared by dissolving PANI in a camphor sulfonic acid/m-cresol solution under sonication, then adding chloroform after filtration. Because we wished to have undoped LB PANI films, we resorted to the strategy of mixing PANI and cadmium stearate. With this mixed monolayer, good-quality Y-type LB films could be produced with a transfer ratio close to 1 . All the experiments were carried out with a KSV 5000 Langmuir trough housed in a class 10,000 clean room. Water for the subphase was supplied by a Millipore Milli-Q system, and the experiments were performed at room temperature. The LB films were deposited on quartz plates, which were chosen as substrates because unlike glass their transparency is kept even after $36 \mathrm{~h}$ of $\mathrm{X}$ ray irradiation, thus preventing possible discrepancies in the UV-VIS. measurements. X-ray exposure was carried out with a Müller MG 150 (Philips) X-ray source at $75 \mathrm{kV}$ and $15 \mathrm{~mA}$. The peak of the irradiation occurs around $0.4 \AA$. A uniform beam with $9.5 \mathrm{~Gy} / \mathrm{s}$ was made to impinge onto the LB film sample that was placed $8 \mathrm{~cm}$ from the source. Irradiations were performed at room temperature in vacuum and with various atmospheres, namely oxygen, nitrogen, argon, and air at different values of relative humidity. The latter was controlled with proper salt amounts inside the closed chamber containing the sample [86]. The relative humidity was measured with an IOPE 10PH-FA-2 hygrometer (Brazil), and X-ray exposure started one hour after a new atmosphere was established in order to stabilize the humidity.

Samples with different numbers of layers were systematically exposed to X-ray irradiation at specific time intervals, with the UV-VIS. spectra acquired right after the X-ray exposure. In all cases there was a shift in the characteristic absorption band of the polymer to higher wavelength values $(>700 \mathrm{~nm})$ with increasing irradiation time, as seen in Fig. 10. The shift in the absorption band is similar to the doping process occurring in thicker polyaniline films [82], but is faster in thinner films and at higher humidity levels $[84,85]$. In order to assess the effects from humidity, 13-layer LB films were exposed to X-ray irradiation during 45 minutes in different atmospheres as illustrated in Fig. 11. In dry atmospheres, such as argon in Fig. 11b, there is a small shift in the band and a decrease in intensity. The same occurs for dry air (relative humidity lower than $2 \%$ ), oxygen and nitrogen. Therefore, PANI in the LB film is not doped under dry atmospheres, consistent with previous results [84]. On the 
other hand, X-ray irradiation at $85 \%$ of relative humidity in argon for the same time period causes the $600 \mathrm{~nm}$ peak to disappear with the concomitant appearance of the characteristic $800 \mathrm{~nm}$ polaronic band (Fig. 11c).

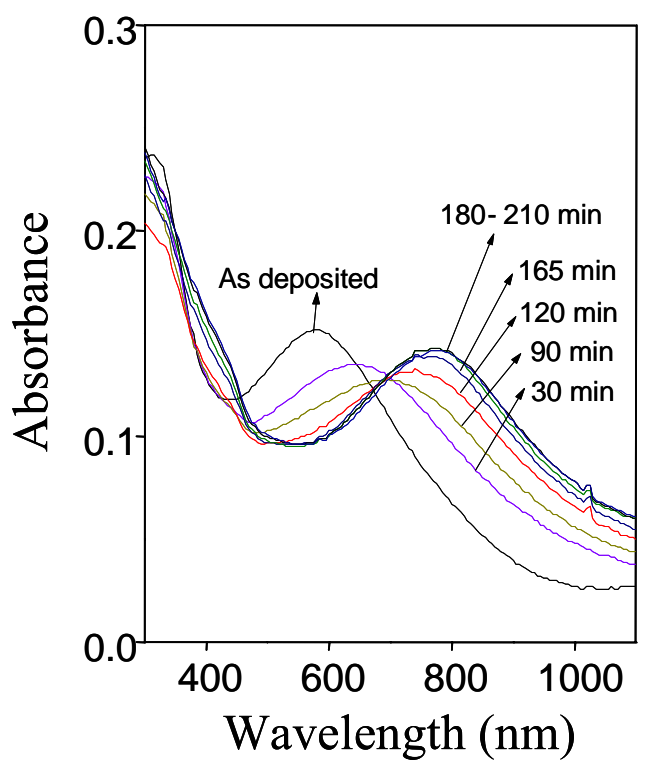

Figure 10. UV-VIS. spectra for a 84-layer LB film subjected to Xray irradiation for increasing periods of time under air with relative humidity of $90 \%$. At the end of the experiments the LB sample had been irradiated for $210 \mathrm{~min}$.

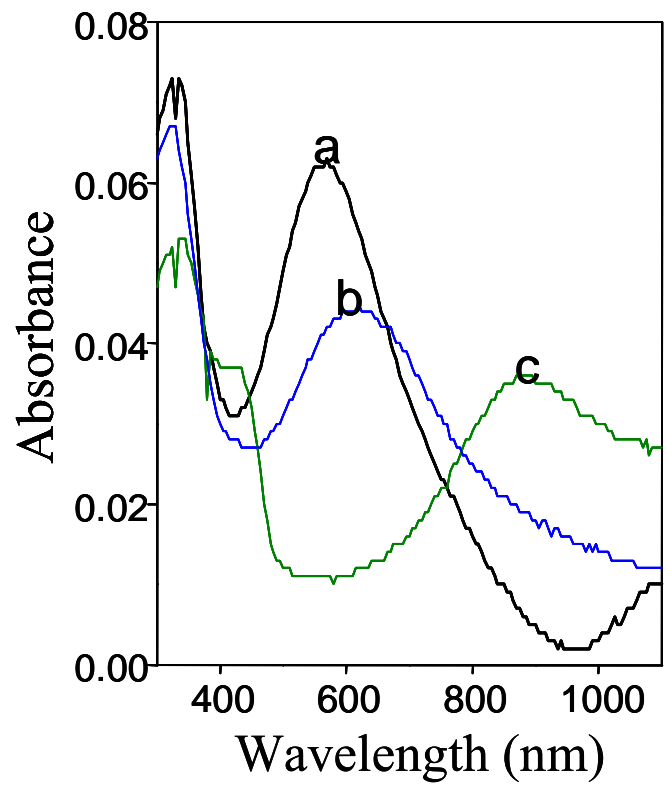

Figure 11. 13-layer composite LB films of polyaniline: a) asdeposited; b) exposed for $45 \mathrm{~min}$ to X-ray irradiation in dry argon atmosphere; c) exposed for 45 min of X-ray irradiation in argon atmosphere at $85 \%$ of relative humidity.

The effects from water in the irradiation-induced doping of PANI can be understood as follows. High-energy particles passing through matter produce ionization, while a drop of water will condense faster on a charged ion than on a neutral ion [89]. Therefore, the higher the humidity level the higher ionization is. The X-ray beam energy used here $\left(\sim 7.10^{5} \mathrm{Kcal} / \mathrm{mol}\right)$ can break water molecules (492 Kcal/mol), $\mathrm{NH}--\mathrm{N}$ hydrogen bonds $(\sim 10 \mathrm{Kcal} / \mathrm{mol})$ [90], and ionize chemical species in the atmosphere studied $(749 \mathrm{Kcal} / \mathrm{mol}$ for oxygen, $853.6 \mathrm{Kcal} / \mathrm{mol}$ for nytrogen, $621 \mathrm{Kcal} / \mathrm{mol}$ for argon, $800 \mathrm{Kcal} / \mathrm{mol}$ for $\mathrm{CO}_{2}$, and 818.8 $\mathrm{Kcal} / \mathrm{mol}$ for dry air [91-93]). There is also the possibility of indirect formation of ionic species due to interactions between X-ray photons and gases or polyaniline molecules. Hence, in addition to ionization by direct excitation with $\mathrm{X}$-rays, there is ionization caused by collisions from secondary electrons. It should also be noted that X-ray irradiation dopes PANI but does not affect the oxidation state of the polymer. If this occurred, polymer degradation would take place, which was not observed in FTIR spectra taken after X-ray exposure [84]. Being a bulk process, the incorporation of water into the polymeric matrix could introduce some of these ionized species (or even "solvated ions" by water molecules) into the polymer backbone, thus stimulating the protonation process. It can also explain the longer times required for X-ray doping since the absorption of water by the polymer is faster than the times involved in this doping mechanism. Similar results are reported by induced positive corona discharge in polyaniline films [94].

\section{Concluding Remarks}

Water at an air interface may display properties that are significantly different from bulk water, particularly if an organic film - such as a Langmuir monolayer - is adsorbed at the interface. The effective dielectric constant, for instance, varies from the value of bulk water to 2-3 at the film/air interface, with the headgroups of the film-forming molecules sitting on a medium with a dielectric constant of 6-7. These changes occur within a very small layer of material, of the order of a few nanometers $(\mathrm{nm})$. The structured water at the film interface allows for enhanced proton conduction through a H-bond network. In this paper we mentioned simplified models that are able to represent the basic mechanisms of proton transfer and can explain the appearance of a critical density for the film molecules, above which increases are observed in the surface potential and lateral conductance. Mention was also made of the significant effects caused by the presence of tiny amounts of guest molecules in the monolayer. At solid interfaces, especially in the cases where a solid substrate is coated with a nanostructured film, adsorbed water plays an essential role, causing the film properties to change drastically. Examples were given of undesirable effects from adsorbed water, as the cause for non-reproducibility of the electrical response of the films. On the other hand, the large effects from adsorbed water may be exploited in useful applications. This was illustrated in the paper by discussing the development of highly-sensitive taste sensors and in the X-ray induced doping of polyaniline Langmuir-Blodgett (LB) films. Interestingly, doping via X-ray irradiation was only effective if 
the polymer surface was unprotected and in a humid environment, which confirms the importance of water adsorbed at the solid/gas interface.

The interfacial effects commented upon here are still treated in a qualitative way in most cases, as modeling the interfaces at the molecular level is a considerable challenge. Nevertheless, understanding the mechanisms by which adsorbed water causes such strong changes in nanostructured film properties is essential for novel applications to be designed. In this context, tremendous opportunities are envisaged with functionalization of solid surfaces by the assembly of organic supramolecular structures. For example, mimicking the remarkable capability of biological systems to convert, transduce, recognize and process diverse and complex sources of information may have a large technological impact.

\section{Acknowledgments}

The authors acknowledge the financial assistance from FAPESP, CNPq and IMMP/MCT.

\section{References}

[1] O. N. Oliveira Jr., PhD Thesis, University of Wales, Bangor, 1990.

[2] I. Langmuir, J. Am. Chem. Soc. 39, 1848 (1917).

[3] K.B. Blodgett, J. Am. Chem. Soc. 57, 1007 (1935).

[4] O.N. Oliveira Jr, M. Raposo, and A. Dhanabalan, LangmuirBlodgett (LB) and Self-assembled (SA) polymeric films, Handbook of Surfaces and Interfaces of Materials, ed. H.S. Nalwa, Vol. 4, pp. 1-63, Academic Press, 2001.

[5] O.N. Oliveira Jr., Braz. J. Phys. 22, 60 (1992).

[6] R.H. Tredgold, Order in Thin Organic Films, Cambridge University Press, Cambridge (1994)

[7] M.C. Petty, Langmuir-Blodgett Films - An Introduction, Cambridge University Press, Cambridge (1996).

[8] G. G. Roberts, Ed. Langmuir-Blodgett Films, Plenum Press, New York, (1990).

[9] G. Decher, J.D. Hong, and J. Schmitt, Thin Solid Films 210/211, 831 (1992).

[10] L. Netzer and J. Sagiv, J. Am. Chem. Soc. 105, 674 (1983).

[11] G. Decher, Science, 277, 1232 (1997).

[12] M. Ferreira and M. Rubner, Macromolecules 28, 7107 (1995)

[13] Y. Lvov, K. Ariga, I. Ichinose, and T. Kunitake, J. Am. Chem. Soc. 117, 6117 (1995).

[14] M. R. Linford, M. Auch, and H. Möhwald, J. Am. Chem. Soc. 120, 178 (1998).

[15] O.N. Oliveira Jr, J.-A. He, V. Zucolotto, S. Balasubramanian, L. Li, H.S. Nalwa, J. Kumar, and S.K. Tripathy, Layer-byLayer Polyelectrolyte Films for Electronic and Photonic Applications, Handbook of Polyelectrolytes, ed. J. Kumar, S.K. Tripathy and H.S. Nalwa, American Scientific Publishers, Los Angeles, Vol. 1, pp. 1-37, 2002.
[16] G.L. Gaines Jr., Insoluble Monolayers at Liquid-Gas Interfaces, Interscience, New York, (1966).

[17] M. Lösche and H. Möhwald, Rev. Sci. Instrum. 55, 1968 (1984).

[18] H. Sakai and J. Umemura, Langmuir 14, 6249 (1998).

[19] X.L. Zhou and S.H. Chen, Phys. Rep. 257, 223 (1995).

[20] D. Ducharme, A. Tessier, and S.C. Russev, Langmuir 17, 7529 (2001).

[21] D.M. Taylor and G.F. Bayes, Mat. Sci. Eng. C. 8, 65 (1999).

[22] H. Morgan, D.M. Taylor, and O.N. Oliveira Jr., Chem. Phys. Lett. 150, 311 (1988).

[23] G.A. Sefler, Q. Du, P.B. Miranda, and Y.R. Shen, Chem. Phys. Lett. 235, 347 (1995).

[24] P. Dynarowicz-Latka, A. Dhanabalan, and O.N. Oliveira Jr., Adv. in Colloid and Interface Sci., 91, 221 (2001).

[25] A. Noblet, H. Ridelaire, and G. Sylin, J. Phys. E: Sci. Instrum. 17, 234 (1984).

[26] A. Cavalli, PhD Thesis, Instituto de Física de São Carlos, Universidade de São Paulo (1997).

[27] D. Ducharme, C. Salesse, and R.M. Leblanc, Thin Solid Films 132, 83 (1985).

[28] R.C. Ahuja, P. Caruso, D. Möbius, G. Wildburg, H. Ringsdorf, D. Philp, J.A. Preece, and J.F. Stoddart, Langmuir 9, 1534 (1993).

[29] A. Dhanabalan, A. Riul Jr., and O.N. Oliveira Jr., Supramolecular Science 5, 75 (1998).

[30] M.I. Mosquéra-Sánchez, PhD Thesis, Instituto de Física de São Carlos, USP (2000)

[31] H. Morgan, D.M. Taylor, and O.N. Oliveira Jr., Biochim. Biophys. Acta 1062, 149 (1991).

[32] F.M. Menger, S.D. Richardson, and G.R. Bromley, J. Am. Chem. Soc. 111, 6893 (1989).

[33] V.L. Shapovalov and Y.V. Ilichev, Chem. Phys. Lett. 197, 303 (1992).

[34] A. Cavalli and O.N. Oliveira Jr., Rev. Sci. Instrum. 66, 5567 (1995).

[35] T. Yoshida, Y. Yamamoto, K. Taga, H. Kamaya, and I. Ueda, J. Phys. Chem. B 107, 3196 (2003)

[36] D.M. Taylor, O.N. Oliveira Jr., and H. Morgan, Thin Solid Films 173, L141 (1989).

[37] W. Caetano, M. Ferreira, M. Tabak, M.I. Mosquéra-Sánchez, O.N. Oliveira Jr, P. Krüger, M. Schalke, and M. Lösche, Biophysical Chemistry 91, 21 (2001).

[38] V. Vogel and D. Möbius, J. Colloid Interf. Sci. 126, 408 (1988).

[39] R.J. Demchack and T.J. Fort Jr., J.Coll. Interf. Sci. 46, 191 (1974).

[40] O.N. Oliveira Jr, D.M. Taylor, T.J. Lewis, S. Salvagno, and C.J.M. Stirling, J. Chem. Soc.: Faraday Trans I, 85, 1009 (1989).

[41] P. Dynarowicz-Latka, A. Cavalli, D.A. Silva Filho, P. Milart, M.C. dos Santos, and O.N. Oliveira Jr., Chem. Phys. Lett. 337, 11 (2001).

[42] G. Gouy, J. Phys. 9, 457 (1910). 
[43] D. M. Taylor, O.N. Oliveira Jr., and H. Morgan, Chem.Phys.Lett. 161, 147 (1989).

[44] P. Dynarowicz-Latka, A. Cavalli, and O.N. Oliveira Jr., Thin Solid Films 360, 261 (2000).

[45] A. Cavalli, P. Dynarowicz-Latka, O.N. Oliveira Jr., and E. Feitosa, Chem. Phys. Lett. 338, 88 (2001).

[46] V.B.P. Leite, A. Cavalli, and O.N. Oliveira Jr., Phys. Rev. E 57, 6835 (1998).

[47] L. Pauling, The Nature of the Chemical Bond. Ithaca, NY: Cornell University Press (1939).

[48] L.C. Allen, J. Am. Chem. Soc. 97, 6921 (1975).

[49] L. Turi and J.J. Dannenberg, J. Phys. Chem. 97, 7899 (1993).

[50] H.-B. Bürgi and J.D. Dunitz, Acc. Chem. Res. 16, 153 (1983).

[51] D. Eisenberg and W. Kauzmann, The Structures and Properties of Water, Oxford, London (1969).

[52] I. Ohmine and S. Saito, Acc. Chem. Res. 32, 741 (1999).

[53] C. Kobayashi, S. Saito, and I. Ohmine, J. Chem. Phys., 13, 9090 (2000).

[54] P.M. Kiefer, V.B.P. Leite, and R.M. Whitnell, Chem. Phys. 194, 33 (1995)

[55] J.F. Tocanne and J. Teissié, Biochim. Biophys. Acta, 1031, 111 (1990).

[56] E. Bosch, M. Moreno, J. Lluch, and J. Bertran, Chem. Phys. 148, 77 (1990).

[57] W. Luck, and T. Wess, J. Molecular Structure 270, 229 (1992).

[58] S. Scheiner, Theochem (v113) J. Mol. Struct. 307, 65 (1994).

[59] D. Bazeia, V.B.P. Leite, B.H.B. Lima, and F. Moraes, Chem. Phys. Lett. 340, 205 (2001).

[60] F. Peñacorada, J. Reiche, S. Katholy, L. Brehmer, and M.L. Rodriguez-Méndez, Langmuir 11, 4025 (1995).

[61] L.J. van der Pauw, Philips Res. Repts. 13, 1 (1958).

[62] J.C. Dyre and T.B. Schrøder, Rev. Mod. Phys. 72, 873 (2000).

[63] R. F. Bianchi, G. F. Leal Ferreira, C. M. Lepienski, and R. M. Faria, J. Chem. Phys. 110, 4602 (1999).

[64] R.H. Tredgold, R. Jones, S.D. Evans, and P.I. Willams, J. Mol. Electron. 2, 147 (1986).

[65] R.H. Tredgold, P. Hodge, Z. Aliadib, and S.D. Evens, Thin Solid Films 210, 4 (1992).

[66] C.J.L. Constantino, A. Dhanabalan, and O.N. Oliveira Jr., Coll. Surf. A 198, 101 (2002).

[67] M. Iwamoto, Y. Yoneda, and A. Fukuda, Jpn. J. Appl. Phys. 31, 3671 (1992)

[68] M. Iwamoto, A. Fukuda, and E. Itoh, J. Appl. Phys. 75, 1607 (1994).

[69] M. Raposo, PhD Thesis, Instituto de Física de São Carlos, USP, 1999.
[70] M.J. Yang, Y. Li, N. Camaioni, G. Casalbore-Miceli, A. Martelli, and G. Ridolfi, Sensor Actuat. B 86, 229 (2002)

[71] L. Grigore and M.C. Petty, J. Mat. Sci. 14, 389 (2003).

[72] S. Mintova and T. Bein, Microporous and Mesoporous Materials 50, 159 (2001).

[73] A.Riul Jr, D.S. dos Santos Jr, K. Wohnrath, R. Di Tommazo, A.C.P.L.F. Carvalho, F.J. Fonseca, O.N. Oliveira Jr, D.M. Taylor, and L.H.C. Mattoso, Langmuir 18, 239 (2002).

[74] A. Riul Jr., R.R. Malmegrim, F.J. Fonseca, and L.H.C. Mattoso, Biosensors \& Bioelectron. 18, 1365 (2003)

[75] A. Riul Jr., A.M.G. Soto, S.V. Mello, S. Bone, D.M. Taylor, and L.H.C. Mattoso, Synth. Metals 132, 109 (2003).

[76] M. Ferreira, A. Riul Jr., K. Wohnrath, F.J. Fonseca, O.N. Oliveira Jr., and L.H.C. Mattoso, Anal. Chem. 75, 953 (2003).

[77] D.M. Taylor and A.G. MacDonald, J. Phys. D: Appl. Phys. 20, 1277 (1987).

[78] A. Riul Jr., R.R. Malmegrim, F.J. Fonseca, and L.H.C. Mattoso, Artificial Organs, 27, 469 (2003).

[79] M. Angelopoulos, A. Ray, A.G. MacDiarmid, and A.J. Epstein, Synth. Metals, 21, 21 (1987).

[80] J.P. Travers, P.L. Guyadec, P.N. Adams, P.J. Laughlin, and A.P. Monkman, Synth. Metals, 69, 229 (1995).

[81] E.S. Matveeva, Synth. Metals, 79, 127 (1996).

[82] J.A. Malmonge and L.H.C. Mattoso, Synth. Metals, 84, 779 (1997).

[83] M. Angelopoulos, J.M. Shaw, W.-S. Huang, and R.D. Kaplan, Mol. Cryst. Liq. Cryst. 189, 221 (1990).

[84] A. Dhanabalan, J.A. Malmonge, A. Riul Jr, R.M. Faria, and O.N. Oliveira Jr, Thin Solid Films 329, 808 (1998).

[85] J.A. Malmonge, A. Dhanabalan, A.Riul Jr, R.M. Faria, and O.N. Oliveira Jr, Synth. Metals 101, 801 (1999).

[86] A. Riul Jr., PhD Thesis, Instituto de Física de São Carlos, USP, 1998.

[87] A. Riul Jr, L.H.C. Mattoso, S.V. Mello, G.D. Telles, and O.N. Oliveira Jr, Synth. Metals 71, 2067 (1995).

[88] L.H.C. Mattoso, A.G. MacDiarmid, and A.J. Epstein, Synth. Metals 68, 1 (1994).

[89] A.H. Compton and S.H. Allison, X-rays in theory and experiment, $2^{\text {nd }}$ edition, D. Van Nostrand Company, Inc., 1954.

[90] L. Pauling, The nature of the Chemical bond, $3^{\text {th }}$ edition, Cornell University, 1960.

[91] H.V. Larson, Phys. Rev. 112, 1927 (1958).

[92] T.E. Bortner and G.S. Hurst, Phys. Rev. 93, 1236 (1954).

[93] Z. Bay and H.H. Seliger, Phys. Rev. 120, 141 (1960).

[94] A.E. Job, J.A. Giacometti, and L.H.C. Mattoso, Appl. Phys. Lett. 72, 3279 (1998). 